\title{
3D Anisotropic Elastoplastic-damage Model and its Application in Simulating the Behavior of Rock Materials
}

\author{
Zhang Junfeng ${ }^{1, a}$, Qi Tao ${ }^{2, b}$ \\ ${ }^{1}$ Institute of Mechanics, the Chinese Academy of Sciences, Beijing 100080, China \\ ${ }^{2}$ Institute of Mechanics, the Chinese Academy of Sciences, Beijing 100080, China \\ azhangjf@imech.ac.cn, bqitao@imech.ac.cn
}

Keywords: anisotropy, elastoplastic-damage, tensor decomposition, rock material

\begin{abstract}
A 3D anisotropic elastoplastic-damage model was presented based on continuum damage mechanics theory. In this model, the tensor decomposition technique is employed. Combined with the plastic yield rule and damage evolution, the stress tensor in incremental format is obtained. The derivate eigenmodes in the proposed model are assumed to be related with the uniaxial behavior of the rock material. Each eigenmode has a corresponding damage variable due to the fact that damage is a function of the magnitude of the eigenstrain. Within an eigenmodes, different damage evolution can be used for tensile and compressive loadings. This model was also developed into finite element code in explicit format, and the code was integrated into the well-known computational environment ABAQUS using the ABAQUS/Explicit Solver. Numerical simulation of an uniaxial compressive test for a rock sample is used to examine the performance of the proposed model, and the progressive failure process of the rock sample is unveiled.
\end{abstract}

\section{Introduction}

Because the heterogeneous textures of geomaterials and the wide existence of joints and cracks in geostructures, the rock materials in geotechnical engineering should be considered to be anisotropic. To understand the inelastic behavior of rock materials, finding out an appropriate constitutive model and approaches for numerical simulation has received considerable attention during the last twenty years. Continuum damage mechanics provides a framework for the development of constitutive equations and damage evolution equations for rock materials. Rock materials containing microcracks and defaults usually exhibit permanent plastic strains as well as the reduction of the stiffness during the loading process. Hence, a coupling elastoplastic damage model is necessary to describe the inelastic softening behaviour of this kind of materials.

In recent years, lots of constitutive models have been constructed according to different damage mechanism concerning with geomaterials and have been used in solving engineering problems. Kawamotto (1988) introduced a second rank symmetric tensor of anisotropic damage to study the deformation and fracturing of discontinuous rock mass [1]. Based on the measured characteristics of random crack distribution on the surface of a rock specimen, a probabilistic analysis method of random anisotropic damage mechanics problems was proposed in literature [2], in which a probabilistic distribution law of damage variables for rock mass is presented as a Beta distribution by using the Monte-Carlo statistical simulation method. Dragon (2000) considered the inelastic response on the macroscale for rock-like materials being resulted from the evolution of inner micro- and meso-cracks accompanied with frictional effects regarding closed cracks for compressive loading, and progressive microcracking produced volumetric dilatancy, which induced anisotropy and further pressure sensitivity. A continuum damage model was also presented accounting for frictional sliding over internal crack surfaces to capture salient features of progressive anisotropic degradation of rock-like materials in literature [3]. Mosler (2004) built a 3D anisotropic elastoplastic damage model using discontinuous displacement fields. To develop the constitutive equations for finite element formulations, an elastoplastic continuum model as well as an anisotropic damage model was 
projected onto a surface leading to traction separation laws. The coupling of both continuum models and the derivation of the corresponding constitutive interface law was also described [4].

In this paper, a 3D anisotropic elastoplastic-damage model is presented to describe the inelastic anisotropic behavior of rock materials, within which the tensor decomposition technique is employed. The stress-strain curves for different directions, which can be obtained from the experiment, are assumed to be different in the normal eigenmodes. Hence, these damage variables in six eigenmodes can form a symmetric damage tensor which stipulates the evaluation of effective elastic modulus of the rock material and an adequate description of the evoluation of damage. The implementation of the proposed model is integrated into the well-known computational environment ABAQUS using the ABAQUS/Explicit Solver. The applicability and the implementation of the model is investigated by means of simulating the failure process of a standard uniaxial compressive test of a rock sample. The result of the numerical simulation shows that it is possible to reproduce most of the observable characteristics of anisotropic behavior and damaged zone in rock materials.

\section{Constitution of 3D Orthotropic Elastoplastic-damage Model}

\subsection{Definition of Damage Tensor}

The hypothesis of strain equivalence indicates that a damaged volume of material under the applied stress $\boldsymbol{\sigma}$ shows the same strain response as the undamaged one submitted to the effective stress $\tilde{\boldsymbol{\sigma}}$ [5]. For 3D anisotropic materials, this statement can be expressed as

$$
\tilde{\varepsilon}=\tilde{C}^{-1} \sigma=C^{-1} \tilde{\sigma}
$$

in which $\boldsymbol{C}$ and $\tilde{\boldsymbol{C}}$ are undamaged and damaged stiffness tensors respectively. Hence, the damage tensor can be written by the damaged and undamaged stiffness tensors as the following form

$$
\boldsymbol{D}=\boldsymbol{I}-\boldsymbol{C}^{-1} \tilde{\boldsymbol{C}}^{-1}
$$

where, $\boldsymbol{I}$ denotes $4^{\text {th }}$ rank unit tensor. The damage tensor is described by the damage variables in the main directions, and it is assumed that, for orthotropic materials, the three damage variables in the main directions are mutually independent. The shear damage variables are determined by the damage variables in the main directions, and the three shear damage variables are independent mutually yet. Thus, just like in literature [6], the damage tensor $\boldsymbol{D}$ is denoted as

$$
\boldsymbol{D}=\left[\begin{array}{cccccc}
\mathrm{D}_{1} & 0 & 0 & 0 & 0 & 0 \\
0 & \mathrm{D}_{2} & 0 & 0 & 0 & 0 \\
0 & 0 & \mathrm{D}_{3} & 0 & 0 & 0 \\
0 & 0 & 0 & 1-\sqrt{\left(1-\mathrm{D}_{1}\right)\left(1-\mathrm{D}_{2}\right)} & 0 & 0 \\
0 & 0 & 0 & 0 & 1-\sqrt{\left(1-\mathrm{D}_{1}\right)\left(1-\mathrm{D}_{3}\right)} & 0 \\
0 & 0 & 0 & 0 & 0 & 1-\sqrt{\left(1-\mathrm{D}_{2}\right)\left(1-\mathrm{D}_{3}\right)}
\end{array}\right]
$$

2.2 Constitutive Equation and Decomposition of the Stiffness Tensor

The generalized Hooke's law for orthotropic materials is expressed as

$$
\boldsymbol{\sigma}=\boldsymbol{C}\left(\boldsymbol{\varepsilon}-\boldsymbol{\varepsilon}^{\mathrm{p}}\right)
$$

where $\varepsilon^{\mathrm{p}}$ is plastic strain tensor. if $\lambda$ and $\overline{\boldsymbol{\varepsilon}}$ are an eigenvalue and the corresponding eigenvector of the stiffness tensor $\boldsymbol{C}$, respectively, then they satisfy the following equation

$C \bar{\varepsilon}=\lambda \bar{\varepsilon}$

The stiffness tensor $\boldsymbol{C}$ can be rewritten as 


$$
\boldsymbol{C}=\sum_{\mathrm{A}=1}^{6} \lambda_{\mathrm{A}} \overline{\boldsymbol{E}}_{\mathrm{A}}
$$

where $\lambda_{\mathrm{A}}$ is the Ath eigenvalue and $\overline{\boldsymbol{\varepsilon}}_{\mathrm{A}}$ is the corresponding eigenvector, $\overline{\boldsymbol{E}}_{\mathrm{A}}=\overline{\boldsymbol{\varepsilon}}_{\mathrm{A}} \cdot \overline{\boldsymbol{\varepsilon}}_{\mathrm{B}}^{\mathrm{T}}$ and $\overline{\boldsymbol{\varepsilon}}_{\mathrm{A}} \cdot \overline{\boldsymbol{\varepsilon}}_{\mathrm{B}}^{\mathrm{T}}=0$ (if $\mathrm{A} \neq \mathrm{B}$ ), then the stress tensor of an orthotropic material can be obtained

$$
\boldsymbol{\sigma}=\sum_{\mathrm{A}=1}^{6} \lambda_{\mathrm{A}} \overline{\boldsymbol{E}}_{\mathrm{A}}\left(\boldsymbol{\varepsilon}-\boldsymbol{\varepsilon}^{\mathrm{p}}\right)
$$

An assumption is made here that this property is unchanged during the damaging process. That is, the orientations of the eigenvectors do not change during loading. Then, the damaged stiffness tensor and the elastoplastic stress-strain relationship in damage state can be defined as

$$
\begin{aligned}
& \boldsymbol{C}=\sum_{\mathrm{A}=1}^{6}\left(1-\mathrm{D}_{\mathrm{A}}\right) \lambda_{\mathrm{A}} \overline{\boldsymbol{E}}_{\mathrm{A}} \\
& \boldsymbol{\sigma}=\sum_{\mathrm{A}=1}^{6}\left(1-\mathrm{D}_{\mathrm{A}}\right) \lambda_{\mathrm{A}} \overline{\boldsymbol{E}}_{\mathrm{A}}\left(\boldsymbol{\varepsilon}-\boldsymbol{\varepsilon}^{\mathrm{p}}\right)
\end{aligned}
$$

2.3 Damage Evolution Coupling Plasticity and Stress Increment

In literature [7], the plastic and damage potential functions were assumed being functions of the thermodynamic forces and the scalar damage variable, namely

$$
\begin{aligned}
& \Phi=\Phi(\boldsymbol{\sigma}, \boldsymbol{K} ; \mathrm{D}) \leq 0 \\
& \Psi=\Psi(\boldsymbol{Y} ; \mathrm{D}) \leq 0
\end{aligned}
$$

in which $\mathrm{D}$ is the isotropic damage variable, $\boldsymbol{K}$ and $\boldsymbol{Y}$ are the thermodynamic conjugate forces for plasticity and damage respectively. The evolution laws characterized the rate of change of the internal variables are also given as

$$
\begin{aligned}
& \dot{\boldsymbol{\varepsilon}}^{\mathrm{p}}=\dot{\gamma}^{\mathrm{p}} \cdot \frac{\partial \Phi}{\partial \boldsymbol{\sigma}} \\
& \dot{\mathrm{D}}^{\mathrm{p}}=\dot{\gamma}^{\mathrm{d}} \cdot \frac{\partial \Psi}{\partial \boldsymbol{Y}}
\end{aligned}
$$

where $\dot{\gamma}^{\mathrm{p}} \geq 0$ and $\dot{\gamma}^{\mathrm{d}} \geq 0$ denote the plastic and damage multipliers, respectively. The explicit form of plastic multiplier $\dot{\gamma}^{\mathrm{p}}$ in terms of the prescribed strain rate was also given in literature [7]. Combining Eq.9 and Eq.11, we can obtain the stress tensor in incremental format in order to be convenient for numerical simulation

$$
\Delta \boldsymbol{\sigma}=\sum_{\mathrm{A}=1}^{6}\left(1-\mathrm{D}_{\mathrm{A}}\right) \lambda_{\mathrm{A}} \overline{\boldsymbol{E}}_{\mathrm{A}}\left(\Delta \boldsymbol{\varepsilon}-\Delta \gamma^{\mathrm{p}} \cdot \frac{\partial \Psi}{\partial \boldsymbol{\sigma}}\right)-\sum_{\mathrm{A}=1}^{6} \Delta \gamma^{\mathrm{d}} \cdot \frac{\partial \Phi}{\partial \boldsymbol{Y}} \cdot \lambda_{\mathrm{A}} \overline{\boldsymbol{E}}_{\mathrm{A}} \boldsymbol{\varepsilon}^{\mathrm{e}}
$$

where $\boldsymbol{\varepsilon}^{\mathrm{e}}$ is the elastic strain tensor.

\section{A Numerical Example}

Based on the proposed model, a finite element code was developed as a user subroutine of the ABAQUS/Explicit Solver. Drucker-Prager yield rule is adopted for the plastic behaviour. The performance of the constitutive model was evaluated by a standard uniaxial compressive test. The parameters of rock sample are summarized in Table 1.

Table 1 Rock Sample Parameters 


\begin{tabular}{|c|c|c|c|c|c|c|c|c|}
\hline $\begin{array}{c}\text { Diameter } \\
{[\mathrm{mm}]}\end{array}$ & $\begin{array}{c}\text { Length } \\
{[\mathrm{mm}]}\end{array}$ & $\begin{array}{c}\text { Density } \\
{\left[\mathrm{Kg} / \mathrm{m}^{3}\right]}\end{array}$ & $\begin{array}{c}\text { Young's } \\
\text { module } \\
{[\mathrm{MPa}]}\end{array}$ & $\begin{array}{c}\text { Poisson's } \\
\text { ratio }\end{array}$ & $\begin{array}{c}\text { Tensile } \\
\text { strengt } \\
\mathrm{h} \\
{[\mathrm{MPa}]}\end{array}$ & $\begin{array}{c}\text { Compressive } \\
\text { strength } \\
{[\mathrm{MPa}]}\end{array}$ & $\begin{array}{c}\text { Frictiona } \\
1 \\
\text { angle } \\
{[\text { degree }]}\end{array}$ & $\begin{array}{c}\text { Cohesive } \\
\text { parameter } \\
{[\mathrm{Pa}]}\end{array}$ \\
\hline 50 & 100 & 2500 & 26500 & 0.23 & 28 & 280 & 40 & 50000 \\
\hline
\end{tabular}

The axial loading on the numerical sample in the simulation is controlled in terms of uniform strain rate. The simulating progressive failure process is shown in Fig.1 (form left to right in order). The result of the numerical simulation shows that it is possible to reproduce most of the observable characteristics of failure behavior and damaged zone in rock materials.
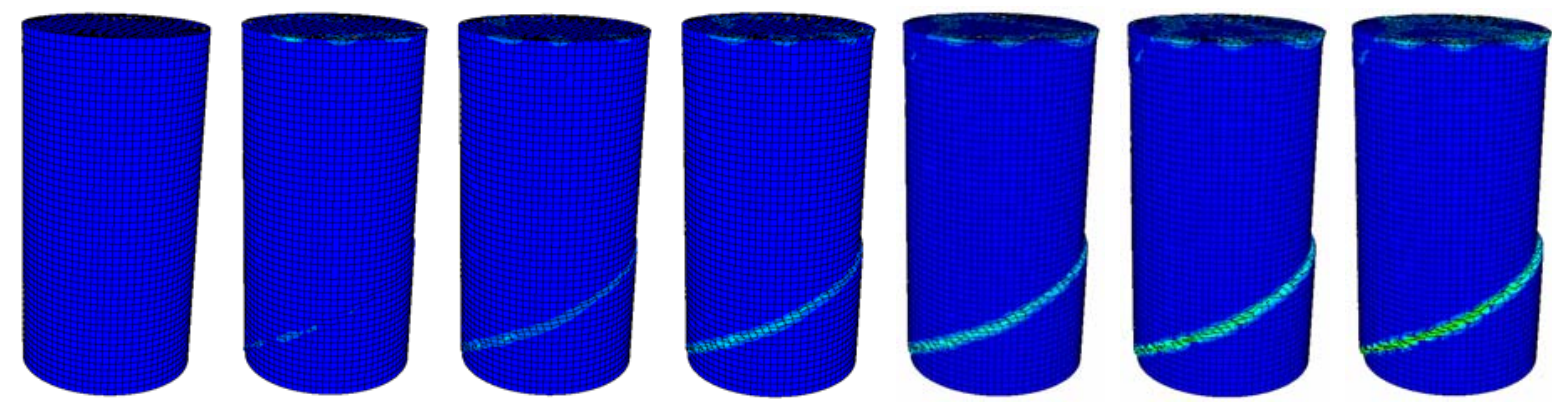

Fig.1 Simulating failure process of a rock sample under uniaxial compression

\section{Summary}

A 3D anisotropic elastoplastic-damage model was presented based on continuum damage mechanics theory. The stress tensor are given in incremental format by using tensor decomposition method. The proposed model is implemented into a finite element computational program ABAQUS/Explicit Solver as its user subroutine. The stress-strain curves for different directions may be different, which can be obtained from the experiment, because of the effect of anisotropy. Different evolution of the damage variables can also be used for tensile and compressive loadings.

\section{Acknowledgements}

This work was supported by the National Natural Science Fundation of China (Grant No.10372104) and the Special Funds for Major State Basic Research Project (Grant No. 2002CB412706).

\section{References}

[1] T. Kawamoto, Y. Ichikawa, and T. Kyoya: Int. J. Numer. Anal. Methods Geomech. Vol.12 (1988), p.1

[2] W.H. Zhang, S. Valliappan: Rock Mech. Rock Engng Vol.23 (1990), p.241

[3] A. Dragon, D. Halm, and Th. Désoyer: Comput. Methods Appl. Mech. Engrg. Vol.183 (2000), p.331

[4] J. Mosler, O. T. Bruhns: Int. J. Numer. Meth. Engng Vol.60 (2004), p.923

[5] J.L. Chaboche: J. Appl. Mech. Vol.55 (1988), p.59

[6] Y. Zhu, S. Cescotto: Int. J. Solids Struct. Vol.32 (1995), p.1607

[7] M.R. Salari, S. Saeb, K.J. Willam, S.J. Patchet, and R.C. Carrasco: Comput. Methods Appl. Mech. Engrg. Vol.193 (2004), p.2625 